\title{
Automatic Segmentation of Brain Structures for Radiation Therapy Planning
}

\author{
Pierre-François D’Haese ${ }^{1,2}$, Valerie Duay ${ }^{1}$, Rui Li $^{1}$, Aloys du Bois d'Aische ${ }^{2}$, Thomas E. Merchant ${ }^{5}$, \\ Anthony J. Cmelak ${ }^{3}$, Edwin F. Donnelly ${ }^{4}$, Kenneth J. Niermann ${ }^{4}$, Benoit Macq ${ }^{2}$, \\ Benoit M. Dawant ${ }^{1}$ \\ ${ }^{1}$ Department of Electrical Engineering and Computer Science, Vanderbilt University, USA \\ ${ }^{2}$ Department of Electrical Engineering, Catholic University of Louvain, Belgium \\ ${ }^{3}$ Department of Radiation Oncology, Vanderbilt University, USA \\ ${ }^{4}$ Department of Radiology and Radiological Sciences, Vanderbilt University, USA \\ ${ }^{5}$ St Jude Children's Research Hospital, Memphis
}

\begin{abstract}
Delineation of structures to irradiate (the tumors) as well as structures to be spared (e.g., optic nerve, brainstem, or eyes) is required for advanced radiotherapy techniques. Due to a lack of time and the number of patients to be treated these cannot always be segmented accurately which may lead to suboptimal plans. A possible solution is to develop methods to identify these structures automatically. This study tests the hypothesis that a fully automatic, atlas-based segmentation method can be used to segment most brain structures needed for radiotherapy plans even tough tumors may deform normal anatomy substantially. This is accomplished by registering an atlas with a subject volume using a combination of rigid and non-rigid registration algorithms. Segmented structures in the atlas volume are then mapped to the corresponding structures in the subject volume using the computed transformations. The method we propose has been tested on two sets of data, i.e., adults and children/young adults. For the first set of data, contours obtained automatically have been compared to contours delineated manually by three physicians. For the other set qualitative results are presented.
\end{abstract}

Keywords: atlas-based segmentation, non-rigid registration, radiation therapy, IMRT

\section{INTRODUCTION}

Radiation has been used to treat cancer since 1898. In the 1960s, medical linear accelerators were developed that could deliver high doses of radiation to deep parts of the anatomy, while sparing superficial tissues. Despite increased energies and better targeting technology, organs and structures adjacent to the cancer still receive large radiation doses. This is the result of a compromise between delivering enough radiation to areas of disease and accepting undesirable "side effects" to normal tissues. There is evidence that even higher radiation doses may cure more cancers, but higher doses are often considered too great a risk with the inaccuracies of conventional radiation therapy planning methods. As powerful computers and tomographic image acquisition methods become more readily available, conformal radiation therapy using intensity modulated radiotherapy with inverse treatment planning (IMRT) has been proposed as a possible solution. The goal of conformal radiation is to have the prescribed radiation dose distribution shaped so as to "conform" to a target volume. New mechanisms for precise beam shaping allow for higher radiation dose to the target (a process known as dose escalation) while keeping the radiation doses to the non-target tissues at acceptable levels. 
Unfortunately, conformal radiation therapy relies on the careful and precise delineation of critical structures on either Computed Tomography (CT) or Magnetic Resonance (MR) images. Typically, this involves delineating by hand structures such as the orbit, lens, optic nerves, optic chiasm, pituitary gland, and brainstem. As dose escalation becomes more common, increasing doses to the precentral gyrus (motor strip), Broca's and Wernicke's speech areas, and primary sensory areas will necessitate the segmentation of these areas as well. At present, 31 centers are treating patients using conformal radiation IMRT therapy techniques. It is estimated that 1000 facilities will begin using these methods within the next 5 to 10 years. Because the potential improvement in the therapeutic ratio provided by conformal methods over conventional radiation delivery techniques is dependent upon precise segmentation and because manual delineation of critical structures is both difficult and time consuming, the lack of automatic segmentation methods will limit the utility of conformal dose delivery in many of these centers.

A number of techniques have been proposed over the years to segment medical images automatically or semiautomatically but for our application which necessitates the segmentation of internal structures and substructures, atlasbased methods are the most appropriate. These methods rely on the existence of a reference image volume (the atlas) in which structures of interest have been carefully segmented, possibly by hand. To segment a new image volume, a transformation that registers the atlas to this volume is first computed. This transformation is then used to project labels assigned to structures from the atlas onto the image volume to be segmented. Techniques that permit the computation of transformations capable of warping one brain onto the other have been proposed over the years (see for instance Collins et al. ${ }^{1}$, Bajcsy et al.2, Christensen et al. ${ }^{3}$, Bro-Nielsen et al. ${ }^{4}$, Meyer et al. ${ }^{\text {Error! Reference source not found. }}$, Rueckert et al. ${ }^{6}$, Woods et al. ${ }^{7}$, or Thirion et al. ${ }^{7}$ ). These methods have been used for the creation of statistical atlases, for the comparison of morphological characteristics between populations, or for segmentation. However, with a few exceptions (Dawant et al. ${ }^{7}$ and Bach et al. ${ }^{8}$ ) these studies have been conducted on image volumes that do not contain tumors which can alter the anatomy of the brain considerably. The objective of the study presented herein is to assess the validity of atlas-based techniques for the automatic segmentation of most structures delineated for typical radiation therapy applications.

\section{METHOD}

\subsection{Data Sets}

The technique we propose has been tested on two different data sets that present different challenges: adults who underwent radiation therapy and children/young adults. The first data set is the least challenging one because tumors are typically small and do not induce large displacements of surrounding structures. The second data set is more challenging because brain morphology is much more variable in children and infants than it is in adults. Some of the subjects included in this data sets also had severe enlargements of the ventricles which did necessitate very large deformations of the atlas. Those last cases are the most challenging ones.

2.1.1 Adults data set: This set consists of 20 adults ( 11 of which were used for quantitative validation). All the image volumes were obtained using a General Electric 1.5-T Signa MR scanner. Each volume consists of 124 sagittal slices, and each slice has dimensions of $256 \times 256$ pixels. Voxel dimensions are $0.94 \times 0.94 \times 1.3 \mathrm{~mm}^{3}$ in the original volumes and each volume was resampled to a 256X256X256 $1 \mathrm{~mm}^{3}$ isotropic volume before processing. Pathologies visible in this data set range from an unresectable glioblastoma multiforme to a prepontine meningioma to a metastasis caused by breast or lung cancer.

2.1.2 Children/young adults data set: This set consists of a set of 45 children to young adults patients (age between 1 and 21) with infratentorial ependymoma. The imaging studies were performed on Siemens Vision and Symphony 1.5T MR systems. Each volume consists of 65 axial slices, and each slice has dimensions of 256x256 pixels. Voxels dimensions are $0.78 \times 0.78 \times 3 \mathrm{~mm}^{3}$. The spatial resolution of this data set is thus lower than the spatial resolution of the first one but each volume was also resampled to a 256X256X256 $1 \mathrm{~mm}^{3}$ isotropic volume before processing. Segmentation has been done for all volumes but at the time of writing only qualitative validation has been performed. 
Figure 1 shows a few slices in representative volumes in the two data sets. The top row illustrates volumes taken from the adult data sets while the bottom row shows volumes from the children/young adults data set.

\subsection{Atlas creation}

\subsubsection{Adults data set.}

The volume used as an atlas was chosen among the volumes used in the study. It was visually selected as being representative of the population (average brain size or shape, average ventricular size, and with only a very small tumor). For each structure to be segmented, a radiation oncologist [AC)] has delineated contours on each individual slice. Delineation was performed either on the sagittal, coronal, or transverse view using a tool developed in-house for this purpose. Because it is difficult for a human operator to draw contours on consecutive slices that lead to smooth 3D shapes, these were postprocessed. To do so, we model the surface of the structures to be segmented as cylindrical surfaces (the opening of a cylindrical surface can be made arbitrarily small such that a close surface can be obtained). These surfaces are

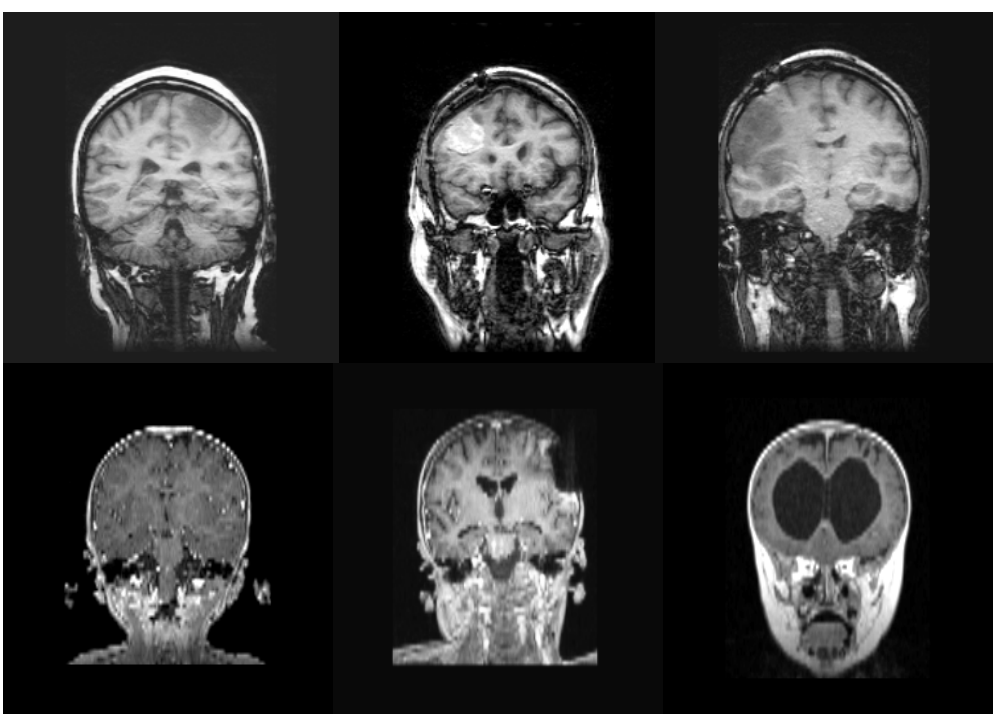

Figure 1: Slices in representative volumes used in this study. Top: adult volumes with left posterior frontal metastases (left), frontal brain metastasis (middle), and unresectable glioblastoma multiform (right). Bottom, three volumes in the children/young adults data set. parameterized and defined as the mapping

$$
\begin{aligned}
& c: \Omega=[0,1] x[0, N-1] \mapsto R^{3} \\
& (s, z) \mapsto c(s, z)=(X(s, z), Y(s, z), z)
\end{aligned}
$$

in which $N$ is the number of slices spanned by the structure and $X(s, z)$ and $Y(s, z)$ are 2D surfaces. Thus, for a fixed zvalue this parameterization represents a closed curve parameterized by $s$. Contours drawn manually on consecutive slices are first aligned with each other, parameterized and stacked. This generates two 2D sets of points $X p(s, z)$ and $Y p(s, z)$. The surfaces $X(s, z)$ and $Y(s, z)$ are computed by fitting smooth surfaces to these sets of points using a smoothing spline approach as proposed by Reinsch ${ }^{10}$.
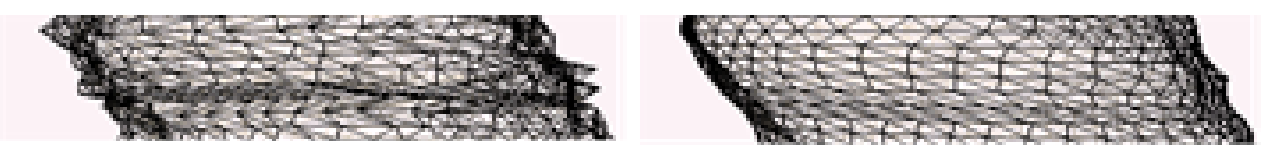

Figure 2 - Contours drawn manually on consecutive slices. Left: prior and right after post-processing. 
In this method parameters for smoothing the surface in the $\mathrm{s}$ direction and in the $\mathrm{z}$ direction can be chosen independently. To preserve the manual contours as much as possible while correcting for contour misalignment when moving from one slice to the other, smoothing was more important in the $\mathrm{z}$ (slice-to-slice direction) than in the $\mathrm{s}$ direction. Values for these parameters were chosen experimentally and varied from one structure to the other. Typically, large structures were smoothed more than smaller ones. Figure 2 shows a cross section of the brainstem before and after post-processing of the contours.

Every atlas structure has been created using the same method from manual contours. Figure 3 shows wire frames of the brainstem, cerebellum, chiasm, pituitary, left and right eyes, lenses and optical nerves. In addition to these structures that were used for validation results, we also delineated the entire cortex for reasons that are explained further.
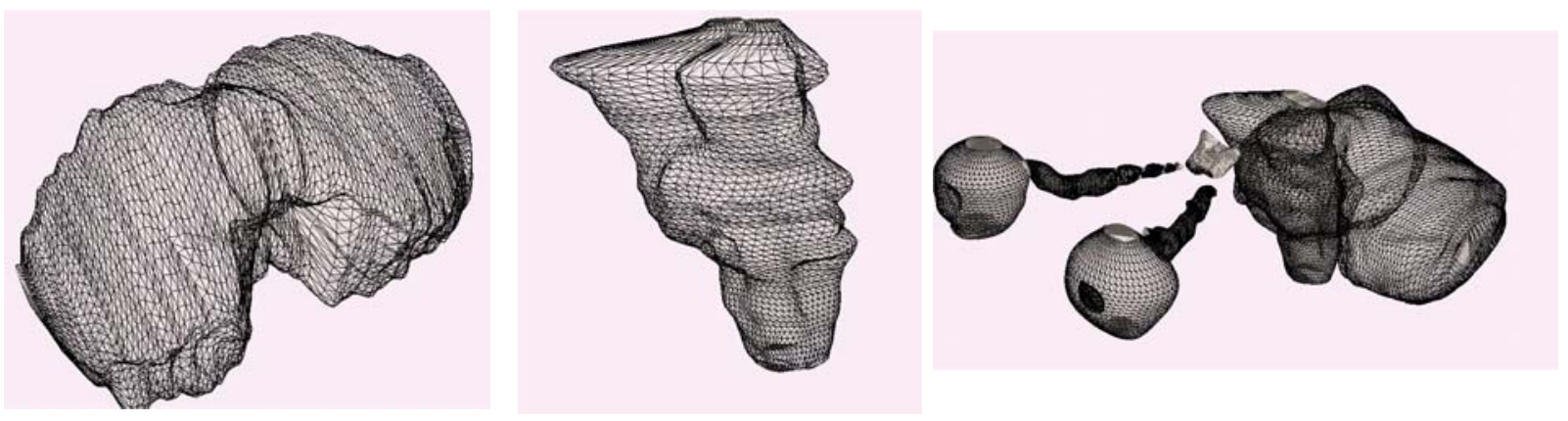

Figure 3: Wire frame representation of the structures defined in the atlas. Left, the cerebellum. Middle, the brainstem, and right the complete set of structures: chiasm, pituitary, optical nerves, cerebellum, eyes and lenses.

2.2.2 Children/young adults data set: The volume used as an atlas was also chosen among the volumes used in the study. It was visually selected as being representative of the population (average brain size or shape, average ventricular size, and presenting all interesting structures). Choosing such a volume for this data set was more challenging because of the large variability in the shape and size of subjects between 1 and 21 years old.

For each structure to be segmented, a radiation oncologist $[\mathrm{TM}]$ has delineated contours on each individual slice. Delineation was performed on the axial slices using the interactive environment we have developed. Contour files were processed as described above to generate smooth 3D surface models in the atlas. For this data set, structures of interest include the brainstem, cerebellum, chiasm, pituitary, and hypothalamus. Other structures such as the left and right thalamus, occipital lobes, meso temporal lobes, temporal lobes, and frontal lobes have been added for testing.

\subsection{Atlas to subject registration}

Registration was performed in two steps. First, the atlas was registered to the volume of interest with a nine degrees of freedom transformation using an independent implementation of a mutual-information based technique similar to the one proposed by Maes et al. ${ }^{11}$. Our implementation permits registration at various resolution levels. In this study, we have used two resolutions $(128 \times 128 \times 128$ and $256 \times 256 \times 256)$ and 64 bins for the reference and target image histograms. In the second step, a non-rigid registration algorithm we have recently proposed was used (Rohde et al. ${ }^{12}$ ). In this technique, inspired by the work of Rueckert et al. ${ }^{5}$ and Meyer et al. ${ }^{13}$, the deformation that registers one image onto the other is modeled with a linear combination of radial basis functions with finite support and the similarity measure used to drive the registration process is the Mutual Information between the images. We have proposed several improvements over existing Mutual Information-based non-rigid registration algorithm ${ }^{\text {Error! Reference source not found., Error! Reference source not found., } 12 .}$ These include working on an irregular grid, adapting the compliance of the transformation locally, decoupling a very large optimization problem into several smaller ones, and deriving schemes to guarantee the topological correctness of the transformations we compute. Our algorithm computes the final deformation field iteratively across scales and resolutions (in this context, resolution means the spatial resolution of the image while the scale is related to the transformation itself). A standard image pyramid is created to apply the algorithm at different resolutions. At each resolution, the scale of the transformation is adapted by modifying the region of support and the number of basis 
functions since the scale of the transformation is related to the bases' region of support (i.e., a large region of support leads to a transformation at a large scale). Typically the algorithm is initialized on a low resolution image with few basis functions having large support. As the algorithm progresses to finer resolutions and smaller scales, the region of support of the basis functions is reduced. Following this approach, the final deformation field is computed as

$$
\mathbf{v}(\mathbf{x})=\mathbf{v}_{1}(\mathbf{x})+\ldots+\mathbf{v}_{M}(\mathbf{x}) .
$$

with $\mathrm{M}$ the total number of levels and one level referring to a particular combination of scale and resolution. Parameters needed by this algorithm were selected on a few volumes and kept fixed for the entire study except for a small number of volumes in the children and young adults data sets with extremely large ventricular enlargement. For these volumes, constraints imposed on the Jacobian of the transformation that guarantee the topological correctness of the transformation had to be relaxed to permit large displacements. The algorithm was applied at two resolutions (64X64X64 and 128X128X128). For our application we did not find any significant improvement when using the full image resolution and we elected not to use it to speed up the process. At the lowest resolution, we used 11 transformation scales (basis functions with region of support ranging from 32 pixels to 5 pixels). At the higher resolution, we use 13 transformation scales (basis functions with region of support ranging from 5 pixels to 2 pixels).

\subsection{Manual contour delineation}

To validate the segmentation results we obtain with our approach, we compared these to contours delineated manually. The procedure we have used to acquire these contours is as follows: for each volume and for each structure, the range of images in which the structure is visible has been manually determined. Fixed numbers (5-8 per structure) of control slices were randomly selected with a uniform distribution within this range. Contours were subsequently drawn manually by three human raters (one is an experienced radiation oncologist [AC], the second one is an experienced radiologist [ED], and the third one $[\mathrm{KN}]$ is a junior physician) on these selected slices. This results in $15-24$ contours (3 times 5-8) per structure for the 11 volumes and 10 structures for a total of 2046 contours ( 3 manual contours on 682 different slices). This procedure permits to test inter-rater variability as well as the similarity between manual and automatic contours. We chose to sample the structures because manual delineation of the entire structures on each volume would have been too time consuming.

\subsection{Projection of the contours delineated on the atlas onto the other volumes}

3D Binary masks were generated from the models obtained with the method described in Section 2.2. These masks were then deformed using the complete deformation fields obtained by composing the two transformations computed to register the atlas to each of the volume. This results in 3D gray-level volumes because of the interpolation required to deform the binary volumes. These gray-level volumes where then threshold at half the intensity value of the masks.

\subsection{Correction of contours}

One area in which the method we propose often leads to poor results is the region around the cerebellum. The main problem we have observed is the inaccuracy of our non-rigid registration algorithm in the lower part of the cerebellum. This is due to a number of factors ranging from poor edge definition to intensity inhomogeneity that affect MR volumes. Because of this, the deformation of the masks defined in the atlas also can lead to inaccurate cerebellum contours. To address this issue we post-process the contours obtained with the atlas-based method. This is done with a geometric deformable model algorithm. In the geometric deformable model framework, the

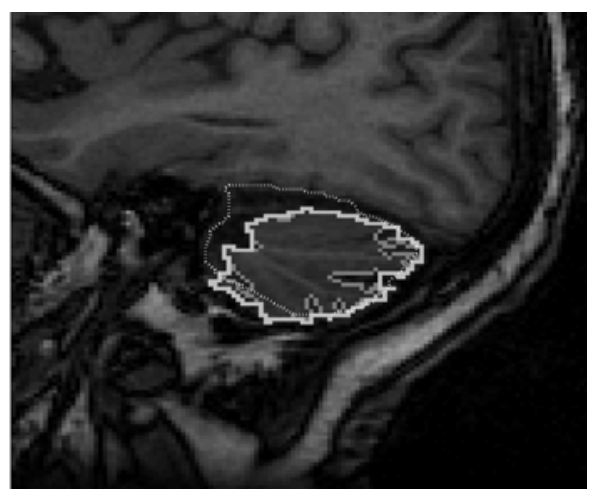

Figure 4: Correction of atlas-based contours. Dotted line: original contours obtained with the atlas; gray line: contours obtained with the level set method; white line: final envelope. evolution of an initial contour toward the true structure boundary is considered as a front propagation problem. This permits the use of level set and fast marching methods introduced by 
Sethian et al. ${ }^{13}$ to model propagating fronts with curvature-dependent speeds. This class of techniques requires two things: (1) an initial boundary and (2) a speed function that specifies the speed at which the boundary evolves along its normal direction. This speed function should be such that it permits the evolution of the boundary toward the edge of the structure and stops its propagation on the edge itself.

To create the initial contours for our application, we erode the atlas-based contours to start as much as possible from inside the cerebellum. To define the speed function, we use several heuristics we have arrived at after observing both the behavior of our algorithm on a large number of image volumes and the image volumes themselves. First, the cerebellum is bounded by the cortex, the brainstem, or cerebrospinal fluid. Second, our atlas-based segmentation algorithm usually produces good results for the cortex and the brainstem. Based on these observations, we have used the following simple speed function:

$$
F(i, j)=\left\{\begin{array}{c}
1 \text { if } x(i, j)>\text { threshold and }(i, j) \notin \Omega \\
0 \quad \text { elsewhere }
\end{array}\right.
$$

In which $\Omega$ is the set of pixels that have been labeled as pertaining to either the brainstem or the cortex following the atlas-based segmentation component of our approach (this is the reason why we have delineated the cortex in the atlas even though we have not defined these contours for each individual subject). This speed function simply says that a contour will expand as long as it travels over a region whose intensity value is above the threshold value and if the pixels over which it travels have not been labeled as either brainstem or cortex pixels. As stated above, it assumes that the initial contour has been placed inside the cerebellum. Note also that the contours produced by this algorithm tend to follow the cerebellum's invagination when the human raters have drawn the cerebellum's envelope. We find the envelope by a morphological closing of the masks obtained with our level set-based contour finding algorithm. Figure 4 shows one example of this process.

\subsection{Validation}

2.7.1 Adults data set. Two methods have been used to compare manual and automatic contours. The first one involves comparing shape and size of the segmented masks, the second compares contours on a point-by-point basis.

Mask comparison: To quantitatively compare contours obtained with the automatic method described above and contours obtained manually, the following similarity measure has been used:

$$
\mathrm{S}=\frac{2 N\left(C_{1} \cap C_{2}\right)}{N\left(C_{1}\right)+N\left(C_{2}\right)}
$$

with $\mathrm{N}\left(\mathrm{C}_{1}\right)$ and $\mathrm{N}\left(\mathrm{C}_{2}\right)$ the number of pixels included in a region, and the manual and automatic contours, respectively. This index ranges from zero to one, with zero indicating no overlap and one indicating a perfect agreement between two contours.

Contour comparison: Mask comparison is a good similarity measure for volumetric measurements but it does not provide precise information on the contours themselves. To gain more insight on this information that is of importance for radiotherapy planning, we also compare manual and automatic contours as follows: from the three manual contours we first compute an envelope as shown in figure 5. This envelope is defined as a band around the structure that encompasses all three manual contours plus one pixel on the inside and one pixel on the outside. We then compute the number of pixels in the automatic contours that fall within this band. We also compute the mean and maximum distance from this band for pixels that fall outside it.

2.7.2 Children/young adults data set: For this data set, manual contours were not available. Validation was performed visually and qualitatively.

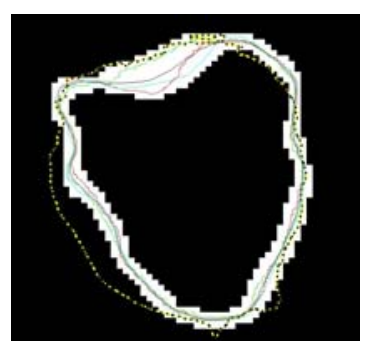

Figure 5: Envelope in white and manual and automatic contours. 


\begin{tabular}{|c|c|c|c|c|c|c|c|c|c|c|c|c|}
\hline & \multirow[t]{2}{*}{$\begin{array}{c}\text { Number } \\
\text { of } \\
\text { Contours }\end{array}$} & \multirow[t]{2}{*}{ Size } & \multicolumn{6}{|c|}{ Sim. Mask Measure (mean values) } & \multicolumn{3}{|c|}{ Contour Similarity } & \multirow[t]{2}{*}{ Perim } \\
\hline & & & A/R1 & $\mathrm{A} / \mathrm{R} 2$ & A/R3 & R1/R2 & R1/R3 & R2/R3 & $\begin{array}{c}\text { In Pts } \\
(\%)\end{array}$ & $\begin{array}{l}\text { mean } \\
(\mathrm{mm})\end{array}$ & $\begin{array}{c}\max \\
(\mathrm{mm})\end{array}$ & \\
\hline Brainstem & 84 & 502 & 0,91 & 0,91 & 0,90 & 0,94 & 0,93 & 0,93 & 0,84 & 1,36 & 2,61 & 102 \\
\hline Cerebellum & 81 & 1264 & 0,87 & 0,87 & 0,87 & 0,92 & 0,92 & 0,94 & 0,72 & 2,19 & 5,23 & 178 \\
\hline Cerebellum (LS) & 75 & 1349 & 0,87 & 0,88 & 0,88 & 0,92 & 0,92 & 0,94 & 0,77 & 2,12 & 4,91 & 183 \\
\hline Left eye & 53 & 462 & 0,91 & 0,89 & 0,86 & 0,92 & 0,89 & 0,89 & 0,83 & 1,09 & 1,66 & 97 \\
\hline Right eye & 54 & 432 & 0,87 & 0,85 & 0,81 & 0,90 & 0,84 & 0,85 & 0,82 & 1,35 & 2,11 & 92 \\
\hline
\end{tabular}

Table 1: Mask and contour-based comparisons between manual and automatic delineation (large structures)

\begin{tabular}{|c|c|c|c|c|c|c|c|c|c|c|c|c|}
\hline & \multirow[t]{2}{*}{$\begin{array}{c}\text { Number } \\
\text { of } \\
\text { Contours }\end{array}$} & \multirow[t]{2}{*}{ Size } & \multicolumn{6}{|c|}{ Sim. Mask Measure (mean values) } & \multicolumn{3}{|c|}{ Contour Similarity } & \multirow[t]{2}{*}{ Perim } \\
\hline & & & $\mathrm{A} / \mathrm{R} 1$ & $\mathrm{~A} / \mathrm{R} 2$ & A/R3 & R1/R2 & R1/R3 & $\mathrm{R} 2 / \mathrm{R} 3$ & $\begin{array}{c}\text { In Pts } \\
(\%)\end{array}$ & $\begin{array}{l}\text { mean } \\
(\mathrm{mm})\end{array}$ & $\begin{array}{c}\max \\
(\mathrm{mm})\end{array}$ & \\
\hline \multirow{2}{*}{$\begin{array}{l}\text { Left lens } \\
\text { Right lens }\end{array}$} & 53 & 34 & 0,56 & 0,55 & 0,56 & 0,77 & 0,80 & 0,80 & 0,60 & 1,35 & 2,11 & 28 \\
\hline & 51 & 38 & 0,69 & 0,70 & 0,70 & 0,81 & 0,81 & 0,85 & 0,76 & 0,97 & 1,28 & 29 \\
\hline \multirow{2}{*}{$\begin{array}{l}\text { Left optical nerve } \\
\text { Right optical } \\
\text { nerve }\end{array}$} & 82 & 28 & 0,51 & 0,53 & 0,49 & 0,69 & 0,71 & 0,67 & 0,57 & 1,82 & 2,96 & 28 \\
\hline & 81 & 29 & 0,59 & 0,60 & 0,60 & 0,72 & 0,68 & 0,68 & 0,67 & 1,03 & 1,62 & 27 \\
\hline Chiasm & 68 & 42 & 0,71 & 0,74 & 0,71 & 0,85 & 0,82 & 0,85 & 0,85 & 0,76 & 0,93 & 41 \\
\hline
\end{tabular}

Table 2: Mask and contour-based comparisons between manual and automatic delineation (small structures)

\begin{tabular}{|c|ccccccccccc|}
\hline & 73 & 76 & 145 & 195 & 281 & 345 & 377 & 381 & 406 & 483 & 632 \\
\hline Brainstem & 0,9 & 0,9 & 0,8 & 0,7 & 0,9 & 0,9 & 0,9 & 0,8 & 0,7 & 0,9 & 1,0 \\
Cerebellum & 0,6 & 0,8 & 0,8 & 0,5 & 0,8 & 0,6 & 0,9 & 0,6 & 0,9 & 0,7 & 0,8 \\
Cerebellum LS & 0,9 & 0,8 & 0,9 & 0,8 & 0,7 & 0,7 & 1,0 & 0,7 & 0,6 & 0,8 & 0,8 \\
Left eye & 0,7 & 0,9 & 0,9 & 0,9 & 0,8 & 0,7 & 0,7 & 0,7 & 0,9 & 1,0 & 0,9 \\
Right eye & 0,8 & 0,8 & 0,8 & 0,9 & 0,8 & 0,9 & 0,7 & 0,8 & 0,8 & 0,9 & 0,9 \\
Left lens & 0,9 & 0,4 & 0,7 & 0,5 & 0,8 & 0,5 & 0,7 & 0,5 & 0,6 & 0,6 & 0,2 \\
Right lens & 1,0 & 0,3 & 0,8 & 1,0 & 0,5 & 0,6 & 0,7 & 0,8 & 0,9 & 0,6 & 0,9 \\
Left optical nerve & 0,5 & 0,4 & 0,7 & 0,7 & 0,5 & 0,7 & 0,5 & 0,7 & 0,3 & 0,7 & 0,7 \\
Right optical nerve & 0,6 & 0,5 & 0,6 & 0,8 & 0,7 & 0,8 & 0,8 & 0,8 & 0,5 & 0,5 & 0,8 \\
Chiasm & 0,7 & 1,0 & 0,8 & 0,9 & 0,8 & 1,0 & 0,8 & 0,9 & 0,7 & 0,9 & 0,9 \\
\hline
\end{tabular}

Table 3: Contour-based comparison results between manual and automatic contours for each subject included in the study.

\section{RESULTS}

\subsection{Adults data set}

Tables 1 and 2 present results we have obtained for the 11 volumes in the first data set for which quantitative validation was performed. These tables show results for the 9 structures (brainstem, cerebellum, chiasm, eyes, lenses and optical nerves) included in the current study. Each table presents values on the two similarity measures discussed in the previous section. The similarity measure based on mask comparison has been computed between each rater (R1 [AC], R2 [ED] and $\mathrm{R} 3[\mathrm{KN}])$ and the atlas $\left(\mathrm{A} / \mathrm{R}_{\mathrm{i}}\right)$, and between each rater for assessment of inter-rater variability $\left(\mathrm{R}_{\mathrm{i}} / \mathrm{R}_{\mathrm{j}}\right)$. For the cerebellum, the table lists two set of numbers obtained before and after (LS) correction with our level-set post processing 
method. The "Size" column lists the average number of pixels in the contours for each structure. The contour similarity measures shows the number of points in the envelope defined as described in the previous section. The second and third columns present the average and maximum distance from the envelope for points falling outside this envelope. The last column provides the average contour perimeter which is a measure of the structure size.

Table 3 lists the contour similarity values for each of the patients included in the study.

\subsection{Children/young adults data}

Figure 6 shows representative segmentation results for the children data set for the axial, sagittal and coronal views. Contours obtained on this data set were only validated visually and qualitatively and were found acceptable. This data set was separated into three subclasses: volumes with small, medium, and large ventricle. This categorization was done because significant ventricular enlargement means large brain deformation which, in turn, challenges atlas-based segmentation method. The three rows in figure 6 show one volume in each category.

The contours shown on these images are the boundaries of ventricles, eyes, frontal, temporal occipital and meso temporal lobes, thalamus, cerebellum, brainstem, chiasm, pituitary and hypothalamus.

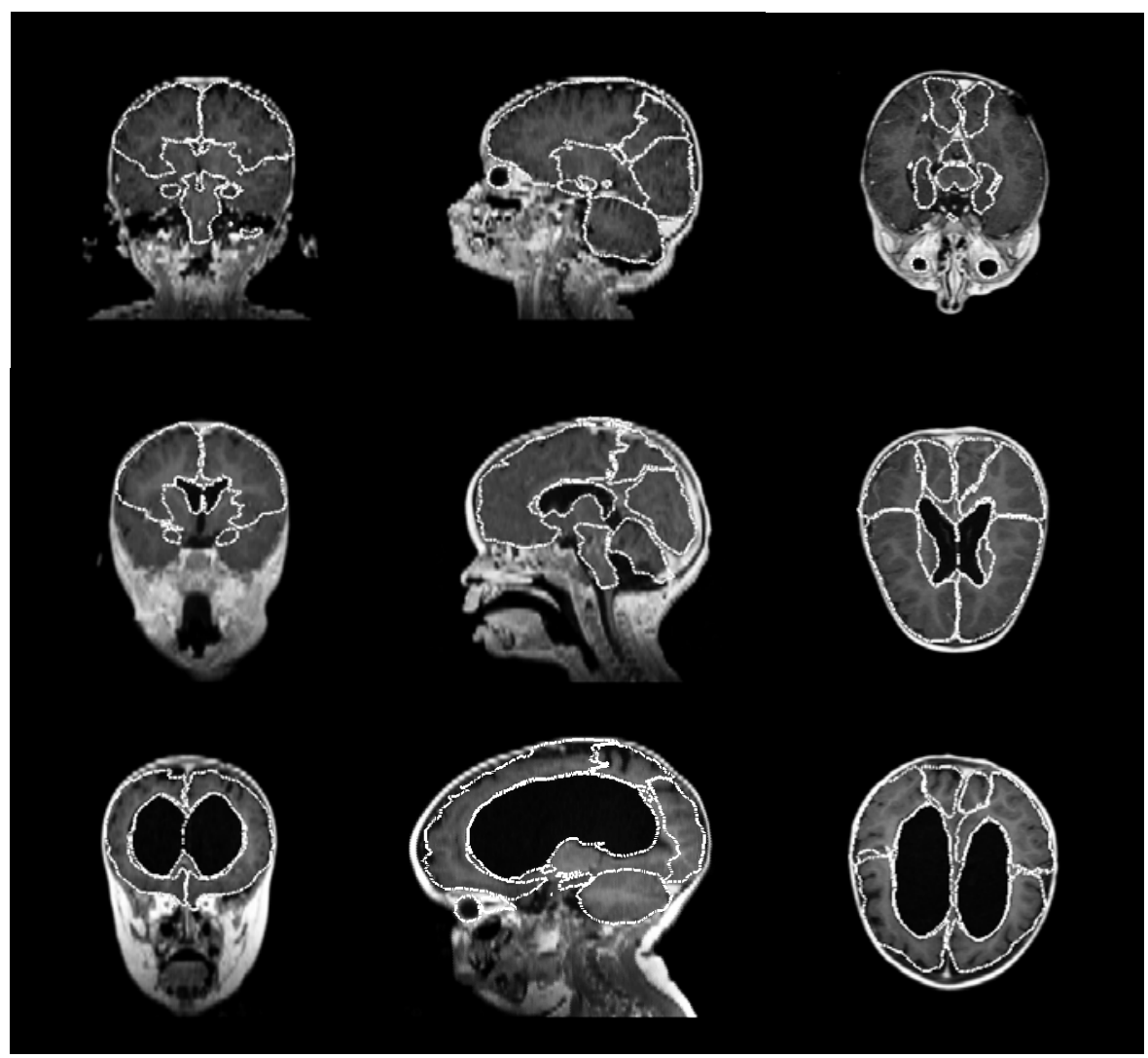

Figure 6: Children segmentation results obtained on the children/young adults data set. From top to bottom one example in the small, medium and large ventricle data sets.

\section{DISCUSSION}

Several conclusions can be drawn both from the quantitative and the qualitative results presented herein. First of all, for relatively large structures such as the eyes, the cerebellum, or the brainstem, the mask-based similarity numbers between manually drawn and automatically obtained contours are very similar. Typically, values of S above 0.8 indicate very good agreement between masks and our study indicates that for these structures, computer-assisted methods are definitely a possibility for contour delineation required for modern radiation-therapy planning. Patient-per-patient study of the results also shows that for these contours obtained manually and contours obtained automatically are very close to each other in the majority of the cases. Results also show that the method we have used to correct for the cerebellar contours does improve things in average but can also negatively impact on the results. The main problem remains the fact that, in the atlas, we have used the envelope of the cerebellum as the region of interest. Based on this convention, all 
three human raters also have delineated the cerebellum's envelope. As shown in figure 7, the envelope is sometimes difficult to locate in a 2D slice. In this figure, the outside gray contour is the contour obtained with our atlas-based method. The other grey contours are the manual ones and the white one is the contour obtained after correction. The corrected contour stopped at the tissue/CSF interface and did not capture the entire envelope as defined by the human raters. We are currently investigating the use of a 3D algorithm to address this issue. The lenses are very small. The average surface of a slice is in the order of $36 \mathrm{~mm}^{2}$ or 36 pixels, for 4 or 5 slices. Often these are not even visible in the images. But by relying on apriori anatomical knowledge, human raters remain able to localize these structures with consistence. Note, however, that even for the manual delineation, the value of the index of similarity is lower for these small structures than it is for the large ones. The optic nerve, while larger than the lenses or the chiasm, is also a challenging structure for purely intensity based methods such as the one we propose. On a portion of its length, it appears as a bright structure surrounded by dark pixels. This good contrast permits a precise registration of the atlas with the volumes to be segmented. On other fractions of its length, the optic nerve is essentially invisible. Again, relying on a-priori anatomic information, human raters are able to deduce the position of the optic nerve both from the position of structures surrounding it and by interpolating mentally its position on slices on which it is invisible from its position on which it is visible. Figure 7 shows several coronal slices in an MR volume in which

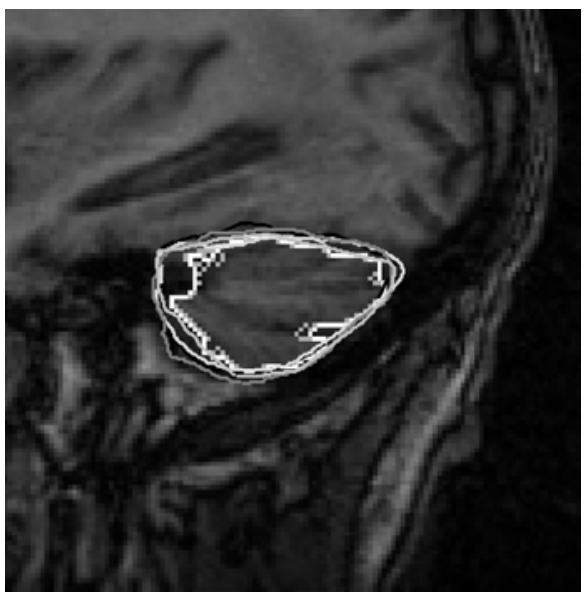

Figure 7: Example of erroneous contour correction. Outside gray contour: atlasbased; other gray contours: manual; white contour: contour obtained with our correction algorithm the optic nerve has been delineated. From left to right, one moves from the chiasm to the eye. On the leftmost panel, the nerve cannot be discerned from its surrounding (the bright rounded structure ion this panel is the carotid artery). On the second panel, the optic nerve is bright and next to the artery. On the third panel it is again difficult to see, and on the two last one if appears as a dark structure surrounded by bright fat. The fact that the images themselves do not carry enough information to permit accurate segmentation suggests complementing our atlas-based approach with a technique in which a-priori shape information can be encoded such as active shape models proposed by Cootes et al. ${ }^{14}$.

Even though quantitative evaluation of the method we propose has not been performed on the children/young adult data set the results we have obtained are very encouraging and warrant further investigation. With deformations and pathologies as large as the ones we have seen in this data set, the validity of an atlas-based approach is questionable. At the

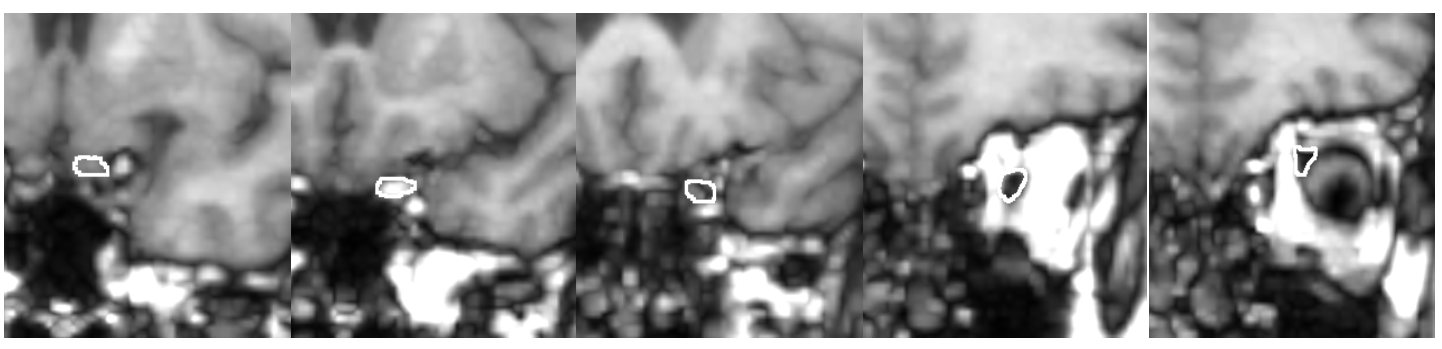

Figure 7: Several coronal slices in an MR volume showing the optic nerve

beginning of the study, we did separate the data set into three categories according to ventricular size as discussed earlier. One atlas was then defined for each category. This was done because it was hypothesized that an atlas with average size ventricles could not be deformed to a subject with very large ventricles. The results we have obtained appear to contradict this initial hypothesis as the contours we have obtained with a single atlas are promising even in the case of severe enlargement and/or pathology.

Even though the quantitative validation we have performed is important, the ultimate objective of this study is to compare radiotherapy plans obtained with the contours obtained automatically to the plans obtained with manual contours. This study is currently ongoing. Should our findings be such that automatic contour delineation can indeed be 
relied upon to produce radiotherapy plans, it could have a significant clinical impact. Complete delineation of the structures included in the adult data set and required for IMRT plans takes over an hour for a trained physician. Even if methods such as the ones we propose are not able to produce accurate contours on each and every slice for every structure of interest, it is highly likely that they will produce acceptable contours on the majority of the slices and require only editing on the others, thus reducing the overall interaction time substantially.

\section{ACKNOLEDGEMENTS}

Pierre-François D'Haese is working towards a Ph.D. degree with awards from FRIA and FNRS (Belgian Science Foundation). This work was supported, in parts by grant NIH/NCI 1R21 CA89657-01A2.

\section{REFERENCES}

1. D. L. Collins, P. Neelin, T. M. Peters, and A. C. Evans, “Automatic 3D intersubject registration of MR volumetric data in standardized Talairach space," J. Comp. Assisted Tomogr. 18(2), pp. 192-205, 1994.

2. R. Bajcsy and S. Kovacic, "Multiresolution elastic matching," Computer Vision, Graphics and Image Processing 46(1), pp. 1-21, 1989.

3. G. E. Christensen, R. D. Rabbit, and M. I. Miller, “3D brain mapping using a deformable neuroanatomy," Physics in Medicine and Biology 39(3), pp. 609-618,1994.

4. M. Bro-Nielsen, and C. Gramkow, "Fast fluid registration of medical images," Proc. Visualization in Biomedical Imaging (VBC'96), pp. 267-276, 1996.

5. Rueckert, L. I. Sonoda, and D. J. Hawkes, "Nonrigid registration using free-form deformations: application to breast MR images," IEEE Transactions on Medical Imaging 18(8), pp. 712-721, August 1999.

6. R. P. Woods, J. C. Mazziotta, and S. R. Cherry, "MRI-PET registration with automated algorithm," J. Comp. Assisted Tomogr., vol. 17, pp. 536-546, July/Aug.1993.

7. J.-P. Thirion, "Image matching as a diffusion process: an analogy with Maxwell's demons," Medical Image Analysis 2(3), pp. 243-260, September 1998.

8. B. M. Dawant, S. L. Hartmann, P. Shiyan, and S. Gadamsetty, "Brain atlas deformation in the presence of large space-occupying lesions,” Journal of Computer Aided Surgery, 7(1):1-10. 2002.

9. M. Bach Cuadra, J. Gomez, P. Hagmann, C. Pollo, J.-G. Villemure, B. M. Dawant, and J.-Ph. Thiran, “Atlas-based Segmentation of Pathological Brains using a Model of Tumor Growth," MICCAI 2002, Tokyo, Japan, pp. 380-387, 2002.

10. C. Reinsch, "Smoothing by spline functions," Numer. Math. 10, pp. 177-183, 1967.

11. F. Maes, A. Collignon, and P. Suetens, "Multimodality image registration by maximization of mutual information," IEEE Transaction on Medical Imaging 16(2), pp. 187-198, April 1997.

12. G. Rhode, A. Aldroubi and B. M. Dawant, "Adaptive-bases algorithm for nonrigid image registration," SPIE Imaging 2002, Image Processing, M. Sonka and J. M. Fitzpatrick (Eds.), Proc. SPIE Vol. 4684, pp.933-944, May 2002.

13. C. R. Meyer et al., "Demonstration of accuracy and clinical versatility of mutual information for automatic multimodality image fusion using affine and thin-plate spline warped geometric deformations," Medical Image Analysis 1(3), pp. 195-206, April 1997.

14. J. A. Sethian, "Recent algorithms for hypersurfaces moving with curvature dependent speed: Hamilton-Jacobi equations and conservation laws," J. Differential Geom., 31, pp. 131-161, 1990.

15. T. F. Cootes, A. Hill, C. J. Taylor and, J. Haslam, "The use of active shape models for locating structures in medical images," Image and Vision Computing 12(6), pp. 355-365, 1994. 